Annals of Pure and Applied Mathematics

Vol. 16, No. 1, 2018, 141-150

ISSN: 2279-087X (P), 2279-0888(online)

Published on 9 January 2018

Annals of

www.researchmathsci.org

DOI: http://dx.doi.org/10.22457/apam.v16n1a16

Pure and Applied

Mathematics

\title{
A Weaker form of Contra Continuous Function in Nano Topological Space
}

\author{
M.Bhuvaneswari $^{l}$ and N.Nagaveni ${ }^{2}$ \\ ${ }^{1}$ Department of Mathematics, Nehru Arts and Science College \\ T.M Palayam, Coimbatore, Tamilnadu, India. E-mail: matjebuvan@gmail.com \\ ${ }^{2}$ Department of Mathematics, Coimbatore Institute of Technology \\ Coimbatore, Tamilnadu, India. E-mail: nagaveni@ cit.edu.in \\ ${ }^{1}$ Corresponding author
}

Received 22 December 2017; accepted 2 January 2018

\begin{abstract}
The purpose of this study is to introduce a weaker form of contra continuous function called Contra Nwg-continuous function in Nano topological spaces. Some of its properties are analyzed. The equivalent condition for a function to be contra Nwgcontinuous function is established. Further Contra Nwg-irresolute function is defined and few of its properties are discussed.
\end{abstract}

Keywords: Nano topological space, Nano continuous function, Nano contra continuous function, $\mathrm{Nwg}$-closed set, $\mathrm{Nwg}$ continuous function, $\mathrm{Nwg}$-irresolute function.

AMS Mathematics Subject Classification (2010): 54A05, 54C10, 54D10

\section{Introduction}

The concept of continuity plays a major role in general topology. Many authors have studied different weaker and stronger form of continuity [11, 12, 17]. Hakawati [7] presented some strong topological aspects in Uryson spaces using closure continuity. In 1994 Dontchev et al [5] introduced the concept of contra-continuity which is a stronger form of LC-continuity in general topological space.

In 2002 [19] Zdzislaw Pawlak discussed the applications of rough set theory with an example. Based on this theory Lellis Thivagar et al [8] defined a new topology called Nano topology in terms of approximations and boundary region of a universal set using equivalence relation on it and studied some weak form of Nano open set. The author [9] established Nano continuity using Nano interior. He [10] also analyzed contra continuous function and bi contra continuous function in nano topological space. Bhuvaneswari et al $[1,2]$ studied various weak form of continuity in Nano topological space. Few other related works in this area can be found in $[6,13,16,18]$.

Nagaveni and Bhuvaneswari $[3,14,15]$ applied the concept of weakly generalization in Nano topology and investigated Nwg-continuous function and Nwgclosed map. The authors [4] have also studied few properties of Nwg-continuous function in terms of Nwg - interior and Nwg - closure. In this paper we introduced weaker forms of different contra continuous functions called Contra Nwg-continuous function and Contra Nwg-irresolute function and discussed few of its characteristics. 


\section{M.Bhuvaneswari and N.Nagaveni}

Throughout this paper $\left(U, \tau_{R}(X)\right)$ is a Nano Topological space with respect to $\mathrm{X}$ Where $X \subseteq U, \mathrm{R}$ is an equivalence relation on $\mathrm{U}, U / R$ denotes the family of equivalence classes of $\mathrm{U}$ by $\mathrm{R}$. $\left(V, \tau_{R^{\prime}}(Y)\right)$ is a Nano Topological space with respect to $\mathrm{Y}$ Where $Y \subseteq V, R^{\prime}$ is an equivalence relation on $\mathrm{V}, V / R^{\prime}$ denotes the family of equivalence classes of $\mathrm{V}$ by $R^{\prime} .\left(W, \tau_{R^{\prime \prime}}(Z)\right)$ is a Nano Topological space with respect

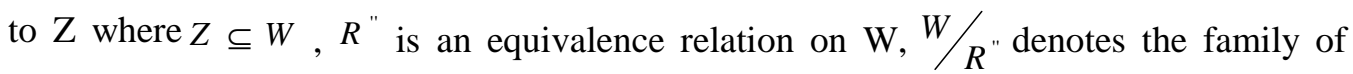
equivalence classes of $\mathrm{W}$ by $R "$.

\section{Preliminaries}

This section is to recall some definitions and properties which are useful in this study.

Definition 2.1. [8] Let $U$ be a non empty finite set of objects called the universe and $R$ be an equivalence relation on $U$ named as the indiscernibility relation. Elements belonging to the same equivalence class are said to be indiscernible with one another. The pair $(\mathrm{U}, \mathrm{R})$ is said to be the approximation space. Let $X \subseteq U$,

1. The Lower approximation of $X$ with respect to $R$ is the set of all objects, which can be for certain classified as $\mathrm{X}$ with respect to $\mathrm{R}$ and is defined by $L_{R}(X)=\bigcup_{x \in U}\{R(x): R(x) \subseteq X\}$. Where $\mathrm{R}(\mathrm{x})$ denotes the equivalence class determined by $\mathrm{x}$.

2. The upper approximation of $X$ with respect to $R$ is the set of all objects, which can be possibly classified as $X$ with respect to $\mathrm{R}$ and is defined by $U_{R}(X)=\bigcup_{x \in U}\{R(x): R(x) \cap X \neq \phi\}$.

3. The boundary region of $X$ with respect to $R$ is the set of all objects, which can be classified neither as $\mathrm{X}$ nor as not- $\mathrm{X}$ with respect to $\mathrm{R}$ and is defined by $B_{R}(X)=U_{R}(X)-L_{R}(X)$.

Definition 2.2. [8] Let $\mathrm{U}$ be the universe, $\mathrm{R}$ be an equivalence relation on $\mathrm{U}$ and $\tau_{R}(X)=\left\{U, \phi, L_{R}(X), U_{R}(X), B_{R}(X)\right\}$ where $X \subseteq U$. Then $\tau_{R}(X)$ satisfies the following axioms.

1. $U$ and $\phi \in \tau_{R}(X)$.

2. The union of the elements of any sub collection of $\tau_{R}(X)$ is in $\tau_{R}(X)$.

3. The intersection of the elements of any finite sub collection of $\tau_{R}(X)$ is in $\tau_{R}(X)$.

That is $\tau_{R}(X)$ forms a topology on $\mathrm{U}$ called as the Nano topology on $\mathrm{U}$ with respect to $X$. We call $\left(U, \tau_{R}(X)\right)$ as the Nano topological space. The elements of $\tau_{R}(X)$ are called as Nano open sets. Elements of $\left[\tau_{R}(X)\right]^{c}$ are called Nano closed sets.

Definition 2.3. [8] If $\tau_{R}(X)$ is the Nano topology on $U$ with respect to $X$, then the set $B=\left\{U, L_{R}(X), U_{R}(X)\right\}$ is the basis for $\tau_{R}(X)$. 
A Weaker form of Contra Continuous Function in Nano Topological Space

Definition 2.4. [8] If $\left(U, \tau_{R}(X)\right)$ is a Nano Topological space with respect to $\mathrm{X}$ where $X \subseteq U$ and if $A \subseteq U$ then the Nano interior of $\mathrm{A}$ is defined as the union of all Nano open subsets of $A$ and it is denoted by NInt(A). Nano interior is the largest Nano open subset of A.

Definition 2.5. [8] The Nano closure of A is defined as the intersection of all Nano closed sets containing $\mathrm{A}$ and it is denoted by $\mathrm{Ncl}(\mathrm{A})$. It is the smallest Nano closed set containing A.

Definition 2.6. [8] Let $\left(U, \tau_{R}(X)\right)$ be a Nano Topological space with respect to $\mathrm{X}$ and $A \subseteq U$. Then A is said to be
(i) Nano semi- open if
$A \subseteq N C l(N I n t(A))$
(ii) Nano pre- open if
$A \subseteq N \operatorname{Nnt}(N C l(A))$
(iii) Nano $\alpha$ - open if
$A \subseteq \operatorname{NInt}(\mathrm{NCl}(\operatorname{NInt}(A)))$
(iv) Nano Regular open if $A=N I n t(N C l(A))$

Definition 2.7. [14] Let $\left(U, \tau_{R}(X)\right)$ be a Nano Topological space. A subset A of $\left(U, \tau_{R}(X)\right)$ is called Nano weakly generalized closed (briefly Nwg-closed) set if $N c l(N \operatorname{int}(A)) \subseteq V$ where $A \subseteq V$ and $\mathrm{V}$ is Nano open. The complement of Nano weakly generalized closed set is Nano weakly generalized open set.

Theorem 2.8. [14] Every Nano open (closed) set is Nano weakly generalized open (closed) set.

Defininition 2.9. The map $f:\left(U, \tau_{R}(X)\right) \rightarrow\left(V, \tau_{R}^{\prime}(Y)\right)$ is called

(i) Nano continuous function [9] if the inverse image of every Nano open set in $V$ is Nano open in U.

(ii) Nano contra continuous function [10] if the inverse image of every Nano open set in $\mathrm{V}$ is Nano closed in $\mathrm{U}$.

(iii) Nwg-continuous function [15] if the inverse image of every Nano open set in V is Nano weakly generalized open in U.

(iv) Nwg-irresolute function [15] if the inverse image of every Nano weakly generalized open set in V is Nano weakly generalized open in $\mathrm{U}$.

Definition 2.10. [4] Nano weakly generalized interior of a subset $A$ is the union of all the Nwg-open sets contained in $\mathrm{A}$.

$\mathrm{NInt}_{\mathrm{wg}}(\mathrm{A})=\cup\{\mathrm{B}: \mathrm{B}$ is Nwg - open sets such that $\mathrm{B} \subseteq \mathrm{A}\}$

Definition 2.11. [4] The intersection of all the Nwg-closed set containing A is called Nwg-closure of A.

$\mathrm{NCl} \underset{\text { wg }}{ }(\mathrm{A})=\cap\{\mathrm{B}: \mathrm{B}$ is $\mathrm{Nwg}-$ closed sets such that $\mathrm{A} \subseteq \mathrm{B}\}$ 
M.Bhuvaneswari and N.Nagaveni

\section{Contra Nwg- continous function}

In this section we define the function called Contra Nwg-continuous function and study some of its properties.

Definition 3.1. The map $f:\left(U, \tau_{R}(X)\right) \rightarrow\left(V, \tau_{R}{ }^{\prime}(Y)\right)$ is Contra Nwg-continuous on $\mathrm{U}$ if the inverse image of every Nano open set in $\mathrm{V}$ is Nano weakly generalized closed set in $\mathrm{U}$.

Example 3.2. Let $U=\{a, b, c, d, e\}$ with $U / R=\{\{a\},\{b\},\{c\}\{d\},\{e\}\}$ and $X=\{a, b\}$. Then the Nano topology is $\tau_{R}(X)=\{U, \phi,\{a, b\}\}$.

Let $V=\{a, b, c, d, e\}$ with $V / R^{\prime}=\{\{d, e\},\{a, c\},\{b\}\}$ and $Y=\{a, b, c\}$. Then the Nano topology is $\tau_{R}{ }^{\prime}(Y)=\{V, \phi,\{a, b, c\}\}$.Define $f:\left(U, \tau_{R}(X)\right) \rightarrow\left(V, \tau_{R}{ }^{\prime}(Y)\right)$ as $f(a)=d, f(b)=e, f(c)=a, f(d)=b, f(e)=c$. Then $\mathrm{f}$ is contra Nwg-continuous function.

Theorem 3.3. Let $f:\left(U, \tau_{R}(X)\right) \rightarrow\left(V, \tau_{R}{ }^{\prime}(Y)\right)$ be a function from $\left(U, \tau_{R}(X)\right)$ to $\left(V, \tau_{R}{ }^{\prime}(Y)\right)$, f is contra Nwg - continuous function if and only if inverse image of every Nano closed set in $\mathrm{V}$ is Nano weakly generalized open in $\mathrm{U}$.

Proof: Let $f:\left(U, \tau_{R}(X)\right) \rightarrow\left(V, \tau_{R}{ }^{\prime}(Y)\right)$ and $\mathrm{B}$ be a Nano closed set in V. Since $\mathrm{f}$ is contra Nwg continuous function $f^{-1}(V-B)=U-f^{-1}(B)$ is Nwg-closed in U. Hence $f^{-1}(B)$ is Nwg-open set in V.

Conversely, let B be a Nano open set in V. By assumption is $f^{-1}(V-B)$ Nwgopen set. $f^{-1}(V-B)=U-f^{-1}(B), f^{-1}(B)$ is Nwg-closed set in U. Hence $\mathrm{f}$ is Contra Nwg- continuous function.

Theorem 3.4. Let $f:\left(U, \tau_{R}(X)\right) \rightarrow\left(V, \tau_{R}^{\prime}(Y)\right)$ be a function from $\left(U, \tau_{R}(X)\right)$ to $\left(V, \tau_{R}{ }^{\prime}(Y)\right)$ then the following conditions are equivalent.

Inverse image of every Nano closed set in $\mathrm{V}$ is Nano weakly generalized open in U.

For $x \in U$ and each nano closed set B in V with $f(x) \in B$ there exists an Nwgopen set in U such that $f(A) \subseteq B$.

Proof: (i) $\rightarrow$ (ii)

Let $\mathrm{B}$ be Nano closed set in $\mathrm{V}$ such that $f(x) \in B, x \in U$. Let $A=f^{-1}(B)$, $x \in A$, and $f(A) \subseteq B$.

(ii) $\rightarrow$ (i) 
A Weaker form of Contra Continuous Function in Nano Topological Space

Let B be any Nano closed in $\mathrm{V}, x \in U, f(x) \in B$.There exists an Nwg open set $U_{x}$ such that $f\left(U_{x}\right) \subseteq B . \quad f^{-1}(B)=\bigcup\left\{U_{x}, x \in f^{-1}(B) \in N O(x)\right\}, f^{-1}(B)$ is Nwg-open in U.

Remark 3.5. Composition of two contra Nwg continuous function need not be contra $\mathrm{Nwg}$ continuous function as shown in the following example.

Example 3.6. Let $U=\{a, b, c, d, e\}$ with $U / R=\{\{a, c\},\{b\},\{d\},\{e\}\}$ and $X=\{a, b\}$. Then the Nano topology is $\tau_{R}(X)=\{U, \phi,\{b\},\{a, c\},\{a, b, c\}\}$.

Let $V=\{a, b, c, d, e\}$ with $V / R^{\prime}=\{\{a, b\},\{c, e\},\{d\}\}$ and $Y=\{a, b\}$. Then the Nano topology is $\tau_{R}{ }^{\prime}(Y)=\{V, \phi,\{a, b\}\}$.

Let $W=\{a, b, c, d, e\}$ with $W / R^{\prime \prime}=\{\{a\},\{b\},\{c\}\{d\},\{e\}\}$ and $Z=\{c, e\}$. Then the Nano topology is $\tau_{R}{ }^{\prime}(Z)=\{W, \phi,\{c, e\}\}$.

Define $f:\left(U, \tau_{R}(X)\right) \rightarrow\left(V, \tau_{R}^{\prime}(Y)\right)$ as $f(a)=c, f(b)=d, f(c)=e, f(d)=a, f(e)=b$ and $g:\left(V, \tau_{R}^{\prime}(Y)\right) \rightarrow\left(W, \tau_{R}{ }^{\prime \prime}(Z)\right)$ as $g(a)=b, g(b)=d, g(c)=c, g(d)=e, g(e)=a$.

Here $\mathrm{f}$ and $\mathrm{g}$ are contra Nwg continous function but their composition is not contra Nwgcontinuous function since $f^{-1}\left(g^{-1}(\{c, e\})\right)=f^{-1}(\{c, d\})=\{a, b\}$ is not Nwg-closed.

Remark 3.7. Contra Nwg-continuous function and Nwg-continuous function are independent.

Example 3.8. Let $U=\{a, b, c, d, e\}$ with $U / R=\{\{a, c\},\{b\},\{d\},\{e\}\}$ and $X=\{a, b\}$. Then the Nano topology is $\tau_{R}(X)=\{U, \phi,\{b\},\{a, c\},\{a, b, c\}\}$.

Let $V=\{a, b, c, d, e\}$ with $V / R^{\prime}=\{\{a\},\{b, d\},\{c, e\}\}$ and $Y=\{c, e\}$. Then the Nano topology is $\tau_{R}{ }^{\prime}(Y)=\{V, \phi,\{c, e\}\}$. Define $f:\left(U, \tau_{R}(X)\right) \rightarrow\left(V, \tau_{R}{ }^{\prime}(Y)\right)$ as $f(a)=c, f(b)=e, f(c)=a, f(d)=d, f(e)=b$. Then $\mathrm{f}$ is Nwg- continuous function. Since $f^{-1}(\{c, e\})=\{a, b\}$ is not Nwg-closed set in $\mathrm{U} f$ is not Contra Nwg-continuous function.

Example 3.9. Let $U=\{a, b, c, d, e\}$ with $U / R=\{\{a, c\},\{b\},\{d\},\{e\}\}$ and $X=\{a, b\}$. Then the Nano topology is $\tau_{R}(X)=\{U, \phi,\{b\},\{a, c\},\{a, b, c\}\}$.

Let $V=\{a, b, c, d, e\}$ with $V / R^{\prime}=\{\{a\},\{b\},\{c, d\},\{e\}\}$ and $Y=\{a, b\}$. Then the Nano topology is $\tau_{R}{ }^{\prime}(Y)=\{V, \phi,\{a, b\}\}$.

Let $f:\left(U, \tau_{R}(X)\right) \rightarrow\left(V, \tau_{R}{ }^{\prime}(Y)\right)$ be a function defined as $f(a)=c, f(b)=d, f(c)=e, f(d)=a, f(e)=b$,

$\mathrm{f}$ then $\mathrm{f}$ is Contra Nwg-continuous function. since $f^{-1}(\{a, b\})=\{d, e\}$ is not Nwg-open $\mathrm{f}$ is not Nwg-continuous function. 
M.Bhuvaneswari and N.Nagaveni

Theorem 3.10. Every Nano contra continuous function is contra Nwg-continuous function.

Proof: Let $f:\left(U, \tau_{R}(X)\right) \rightarrow\left(V, \tau_{R}{ }^{\prime}(Y)\right)$ be a Nano contra function from $\left(U, \tau_{R}(X)\right)$ to $\left(V, \tau_{R}{ }^{\prime}(Y)\right)$ and $\mathrm{A}$ be Nano open set in $\mathrm{V}$. Since $\mathrm{f}$ is Nano contra function $f^{-1}(A)$ is Nano-closed set in $\mathrm{U}$. Therefore $f^{-1}(A)$ is Nwg-closed in U.Hence $\mathrm{f}$ is contra Nwgcontinous function.

Remark 3.11. If $f:\left(U, \tau_{R}(X)\right) \rightarrow\left(V, \tau_{R}^{\prime}(Y)\right)$ is a Contra Nwg-continuous function then $\mathrm{f}$ need not be Nano contra continuous function as shown in the following example.

Example 3.12 Let $U=V=\{1,2,3,4,5,6,7,8,9\}$

$$
\begin{aligned}
& R=\{\{x, y\} / x \text { and } y \text { are both even or odd } x, y \in U\} \\
& U / R=\{\{2,4,6,8\},\{1,3,5,7,9\}\}, X=\{2,4,6,8,7,9\}
\end{aligned}
$$

The Nano topology is $\tau_{R}(X)=\{U, \phi,\{2,4,6,8\},\{1,3,5,7,9\}\}$.

Let $R^{\prime}=\{(x, y) / x-y$ is divisible by 6 and $x, y \in V\}, \quad V / R^{\prime}=\{\{1,7\},\{2,8\},\{3,9\}\}$, $Y=\{1,8\}$ then the Nano topology induced by R' is $\tau_{R}^{\prime}(Y)=\{V, \phi,\{1,2,7,8\}\}$.

Let $f:\left(U, \tau_{R}(X)\right) \rightarrow\left(V, \tau_{R}{ }^{\prime}(Y)\right)$ be an identity map from $\mathrm{U}$ to $\mathrm{V}$. Then $\mathrm{f}$ is Contra Nwg-continuous function but not Contra continuous function since $f^{-1}(\{1,2,7,8\})$ is Nwg-closed but not Nano closed.

Theorem 3.13. A function $\mathrm{f}$ from Nano topological space $\mathrm{U}$ to Nano topological space $\mathrm{V}$ is Contra Nwg-continuous function if the only Nano open set containing the inverse image of every Nano open A of $\mathrm{V}$ is $\mathrm{U}$.

Proof: Let $\mathrm{A}$ be a Nano open set of $\mathrm{V}$ and $\mathrm{U}$ is the only Nano open set such that $f^{-1}(A) \subseteq U$. Then $N c l\left(N\right.$ int $\left.\left(f^{-1}(A)\right)\right) \subseteq U$. (i.e) $f^{-1}(A)$ is Nwg-closed in U. $\mathrm{f}$ is Contra Nwg-continuous function.

Corollary 3.14. Let $\mathrm{f}$ be a function defined from Nano topological space $\left(U, \tau_{R}(X)\right)$ to Nano topological space $\left(V, \tau_{R}{ }^{\prime}(Y)\right)$ and $N \operatorname{int}\left(f^{-1}(A)\right)=\phi$ for every Nano open set A of $\mathrm{V}$ then $\mathrm{f}$ is Contra Nwg-continuous function.

Theorem 3.15. Let $\mathrm{f}$ be a contra Nwg-continuous function then $f\left(\right.$ NInt $\left._{\text {wg }}(\mathrm{A})\right) \subseteq N C l(f(A))$ for every subset $A \subseteq U$.

Proof: Let $A \subseteq U$ then $N C l(f(A))$ is a Nano closed set in V. Since $\mathrm{f}$ is contra Nwg-continous $f^{-1}(\operatorname{NCl}(f(A)))$ is Nwg-open set in $\mathrm{U}$ and $\mathrm{NInt}_{\mathrm{wg}}\left(f^{-1}(N C l(f(A)))\right)=f^{-1}(N C l(f(A))) . \quad f(A) \subseteq N C l(f(A))$, 
A Weaker form of Contra Continuous Function in Nano Topological Space

$\mathrm{NInt}_{\mathrm{wg}}(A) \subseteq \mathrm{NInt}_{\mathrm{wg}}\left(f^{-1}(N C l(f(A)))\right), \mathrm{NInt}_{\mathrm{wg}}(A) \subseteq f^{-1}(N C l(f(A)))$, $f\left(\mathrm{NInt}_{\mathrm{wg}}(A)\right) \subseteq N C l(f(A))$.

Remark 3.16. The converse of the above theorem need not be true as shown in the following example.

Example 3.17. In example $3.8 f\left(\mathrm{NInt}_{\mathrm{wg}}(\mathrm{A})\right) \subseteq N C l(f(A))$ but $\mathrm{f}$ is not contra Nwgcontinuous function

Remark 3.18. In theorem 3.15, if $f(A)$ is Nano closed set then $f\left(\mathrm{NCl}_{\text {wg }}(\mathrm{A})\right)=\operatorname{NCl}(f(A))$.

Corollary 3.19. Let $f:\left(U, \tau_{R}(X)\right) \rightarrow\left(V, \tau_{R}^{\prime}(Y)\right)$ be a Nwg-continuous function then $\left.f\left(\mathrm{NInt}_{\text {wg }}\left(\mathrm{f}^{-1}(\mathrm{~A})\right)\right) \subseteq N C l(A)\right)$ for every subset $A \subseteq V$.

Theorem 3.20. Let $f:\left(U, \tau_{R}(X)\right) \rightarrow\left(V, \tau_{R}^{\prime}(Y)\right)$ be a Nwg-continuous function then $f^{-1}(N \operatorname{Int} A) \subseteq N C l_{w g}\left(f^{-1}(A)\right)$ for every subset $A \subseteq V$.

Proof: Let $A \subseteq V$ then NIntA is a Nano open set in V. Since $\mathrm{f}$ is contra Nwgcontinous $f^{-1}(N \operatorname{Int} A)$ is Nwg-closed set in $\mathrm{U}$ and $N C l_{w g}\left(f^{-1}(N \operatorname{Int} A)\right)=f^{-1}(N \operatorname{Int} A)$. $N \operatorname{Int} A \subseteq A, f^{-1}(N \operatorname{Int} A) \subseteq f^{-1}(A), N C l_{w g}\left(f^{-1}(N \operatorname{Int} A)\right) \subseteq N C l_{w g}\left(f^{-1}(A)\right)$, $\left(f^{-1}(N \operatorname{Int} A)\right) \subseteq N C l_{w g}\left(f^{-1}(A)\right)$.

Remark 3.21. The converse of the above theorem need not be true as shown in the following example.

Example 3.22. In example 3.9, $\mathrm{f}$ is not Contra Nwg- continuous function, but $f^{-1}(N \operatorname{Int} A) \subseteq N C l_{w g}\left(f^{-1}(A)\right)$ for every subset $A \subseteq V$.

Remark 3.33. In theorem 3.20, $f^{-1}(N \operatorname{Int} A)=N \operatorname{Int}_{w g}\left(f^{-1}(A)\right)$ if $A$ is Nano open.

\section{Contra Nwg irresolute function}

In this section we introduce Contra Nwg- irresolute function and discuss some of its properties.

Definition 4.1. The map $f:\left(U, \tau_{R}(X)\right) \rightarrow\left(V, \tau_{R}^{\prime}(Y)\right)$ is Contra Nwg irresolute function on $U$ if the inverse image of every Nano weakly generalized open (closed) set in $\mathrm{V}$ is Nano weakly generalized closed (open) in $\mathrm{U}$. 
Example 4.2. Let $U=\{a, b, c, d, e\}$ with $U / R=\{\{a\},\{b\},\{c, d\},\{e\}\}$ and $X=\{c, d, e\}$. Then the Nano topology is $\tau_{R}(X)=\{U, \phi,\{c, d, e\}\}$.

Let $V=\{a, b, c, d, e\}$ with $V / R^{\prime}=\{\{a\},\{b, c\},\{d, e\}\}$ and $Y=\{d, e\}$. Then the Nano topology is $\tau_{R}{ }^{\prime}(Y)=\{V, \phi,\{d, e\}\}$. Define $f:\left(U, \tau_{R}(X)\right) \rightarrow\left(V, \tau_{R}{ }^{\prime}(Y)\right)$ as $f(a)=d, f(b)=e, f(c)=b, f(d)=c, f(e)=a$. Then $\mathrm{f}$ is contra Nwg irresolute function.

Remark 4.3. Composition of two contra Nwg- irresolute function on U need not be contra Nwg-irresolute function as given in the following example.

Example 4.4. Let $U=\{a, b, c, d, e\}$ with $U / R=\{\{a, b\},\{c, d\},\{e\}\}$ and $X=\{c, d, e\}$. Then the Nano topology is $\tau_{R}(X)=\{U, \phi,\{c, d, e\}\}$.

Let $V=\{a, b, c, d, e\}$ with $V / R^{\prime}=\{\{a\},\{b, c\},\{d\},\{e\}\}$ and $Y=\{d, e\}$. Then the Nano topology is $\tau_{R}{ }^{\prime}(Y)=\{V, \phi,\{d, e\}\}$.

Let $W=\{a, b, c, d, e\}$ with $\left.\left.W / R^{\prime \prime}=\{\{a\},\{b\},\{c\} d\}\right\},\{e\}\right\}$ and $Z=\{a, b, d\}$. Then the Nano topology is $\tau_{R}{ }^{\prime}(Z)=\{W, \phi,\{a, b, d\}\}$. Define $f:\left(U, \tau_{R}(X)\right) \rightarrow\left(V, \tau_{R}^{\prime}(Y)\right)$ as $f(a)=d, f(b)=e, f(c)=b, f(d)=c, f(e)=a$

let $g:\left(V, \tau_{R}{ }^{\prime}(Y)\right) \rightarrow\left(W, \tau_{R^{\prime}}{ }^{\prime \prime}(Z)\right)$ as $g(a)=a, g(b)=b, g(c)=d, g(d)=c, g(e)=e$. Here $\mathrm{f}$ and $\mathrm{g}$ are contra Nwg -irresolute functions but their composition is not contra Nwg-irresolute function since $f^{-1}\left(g^{-1}(\{c, e\})\right)=f^{-1}(\{d, e\})=\{a b\}$ is not Nwg-open set in $\mathrm{U}$.

Theorem 4.5. Let $f:\left(U, \tau_{R}(X)\right) \rightarrow\left(V, \tau_{R}^{\prime}(Y)\right), g:\left(V, \tau_{R^{\prime}}(Y)\right) \rightarrow\left(W, \tau_{R^{\prime \prime}}(Z)\right)$ be two contra Nwg-irresoulute functions, then their composition is Nwg - irresolute function.

Proof: Let A be a Nwg-open set in W. $g^{-1}(A)$ is Nwg-closed set in V, since g is contra Nwg-irresolute function. $f^{-1}\left(g^{-1}(A)\right)$ is Nwg-open in $\mathrm{U}$ because $\mathrm{f}$ is contra Nwgirresolute. Hence the composition of $\mathrm{f}$ and $\mathrm{g}$ is $\mathrm{Nwg}$-continous function.

Theorem 4.6. Every Contra Nwg-irresolute function is contra Nwg-continuous function.

Proof: Let $f:\left(U, \tau_{R}(X)\right) \rightarrow\left(V, \tau_{R}{ }^{\prime}(Y)\right)$ be a contra Nwg-irresolute function, and A be a Nano closed set in $\left(V, \tau_{R}{ }^{\prime}(Y)\right)$. A is Nwg-closed set since every Nano closed set is Nwg-closed set. Then $f^{-1}(A)$ is Nwg-open set. Hence $\mathrm{f}$ is contra Nwg-continuous function.

Remark 4.7. Every Contra Nwg- continuous function need not be contra Nwgirresolute function as shown in the following example. 
A Weaker form of Contra Continuous Function in Nano Topological Space

Example 4.8. Let $U=\{a, b, c, d, e\}$ with $U / R=\{\{a\},\{b\},\{c, d\},\{e\}\}$ and $X=\{c, d, e\}$. Then the Nano topology is $\tau_{R}(X)=\{U, \phi,\{c, d, e\}\}$.

Let $V=\{a, b, c, d, e\}$ with $V / R^{\prime}=\{\{a\},\{b\},\{c\}\{d, e\}\}$ and $Y=\{b, c\}$. Then the

Nano topology is $\tau_{R}{ }^{\prime}(Y)=\{V, \phi,\{b, c\}\}$. Define $f:\left(U, \tau_{R}(X)\right) \rightarrow\left(V, \tau_{R}{ }^{\prime}(Y)\right)$ as $f(a)=d, f(b)=c, f(c)=e, f(d)=a, f(e)=b$. Then $\mathrm{f}$ is contra Nwg continuous function but not Contra Nwg-irresolute function since inverse image of Nwg-open set $\{a, b, e\}$ is not Nwg closed in V.

\section{Conclusion}

In this paper, we introduced a weaker form of Contra continuous function called Contra Nwg-continuous function in Nano topological space. Some of its characterization are analyzed in terms of Nwg-closure and Nwg-interior .Various conditions for a function to be Contra Nwg-continuous function is also established. In Section 4 Contra Nwgirresolute function has been introduced and studied few of its properties.

Acknowledgement. The authors would like to thank reviewer(s) of this article for the time they spent and for their valuable suggestions which improved the presentation of the work.

\section{REFERENCES}

1. K.Bhuvaneswari and K.Mythili Gnanapriya, On nano generalised continuous function in nano topological space, International Journal of Mathematical Archive, 6(6) (2015) 182.

2. K.Bhuvaneswari and A.Ezhilarasi, Nano semi-generalized continuous maps in nano topological space, International Research Journal of Pure Algebra, 5(9) (2015) 149155

3. M. Bhuvaneswari and N. Nagaveni, A weaker form of a closed map in nano topological space, International Journal of Innovation in Science and Mathematics, 5(3) (2017) 77-82.

4. M.Bhuvaneswari and N.Nagaveni, On closure operator in Nano topological spaces(communicated)

5. J.Dontchev, Contra continuous functions and strongly S-closed space, International Journal of Math and Sci., 19 (1996) 303-310.

6. M.K.Ghosh, Separation axioms and graphs of functions in nano topological spaces via nano $\beta$-open sets, Annals of Pure and Applied Mathematics, 14(2) (2017) 213223.

7. A.A.Hakawati and M.Abu-Eideh, On strong topological aspects in Uryson spaces, Annals of Pure and Applied Mathematics, 16(1) (2018) 117-125.

8. M.Lellis Thivagar and C.Richard, On nano forms of weakly open sets, International Journal of Mathematics and Statistics Invention, 1(1) (2013) 31-37.

9. M.Lellis Thivagar and C.Richard, On nano continuity, Math. Theory Model., 7 (2013) 32-37.

10. M.Lellis Thivagar, Saeid Jafari and S. Devi, On new class of contra continuity in nano topology, Italian Journal of Pure and Applied Mathematics, 41 (2017) 1-12. 
M.Bhuvaneswari and N.Nagaveni

11. N.Levine, Semi-open sets and semi continuity in topological spaces, Amer. Math. Monthly, 70 (1963) 36-41.

12. A.S.Mashhour, M.E. Abd El-Monsef and S.N. El-Deeb, On pre continuous and weak pre continuous mappings, Proc. Math. Phys. Soc. Egypt, 51 (1982) 47-53.

13. Mohammed M.Khalaf and Kamal N.Nimer, Nano Ps-open sets and Ps-continuity, International Journal of Contemporary Mathematical Sciences, 10 (2015) 1-11.

14. N.Nagaveni and M.Bhuvaneswari, On nano weakly generalized closed set, International Journal of Pure and Applied Mathematics, 106(7) (2016) 129-137.

15. N.Nagaveni and M.Bhuvaneswari, On nano weakly generalized continuous functions, International Journal of Emerging Research in Management \&Technology, 6(4) (2017) 95-100.

16. A.A.Nasef, A.I.Aggour and S.M.Darwesh, On some classes of nearly open sets in nano topological spaces, Journal of Egyptian Mathematical Society, 24 (2016) 585589.

17. I.L.Reilly and M.K Vamanamurthy, On $\alpha$-continuity in topological spaces, Acta Math. Hunga, 45 (1-2) (1985) 27-32.

18. S.B.Shalini, G. Sindhu and K.Indirani, On generalized $\beta$ regular spaces and nano generalized $\beta$ normal spaces in nano topological spaces, Annals of Pure and Applied Mathematics, 14(2) (2017) 225-229.

19. Z.Pawlak, Rough set theory and its applications, Journal of Telecommunications and Information Technology, 3 (2002) 7-10. 